\title{
The reliability analysis for main fuel accelerated regulation system of aero-engine based on interval model
}

\author{
ZHANG Lei-lei ${ }^{1,}$, , LIU Sheng-zhi ${ }^{2, b}$, ZHANG Zheng ${ }^{1, c}$ and ZHANG Feng ${ }^{1, d}$ \\ ${ }^{1}$ Northwestern Polytechnical University, China \\ ${ }^{2}$ AVIC Landing Gear Advanced Manufacturing Corp,China \\ a18710495493@163.com, dnwpuwindy@mail.nwpu.edu.cn
}

\begin{abstract}
Keywords: Main fuel accelerated regulation system; Interval theory; Fault tree; Failure probability; Monte Carlo method

Abstract: To effectively assess the reliability of aero-engine main fuel accelerated regulation system under poor failure statistics information, a fault tree analysis method based on interval model is presented. Firstly, according to main fuel accelerated regulation system principle and failure mechanism, the system fault tree model is established. Then interval number is introduced to describe the uncertainty of bottom event, and achieve the uncertainty transfer from bottom events to top event through constructing AND/OR gate interval operator. Finally, the failure probability estimation process of system top event is proposed based on double Monte Carlo method, and get the accurate failure interval of system response. At the same time, the importance measurement analysis of bottom events is conducted to identify the key failure events, which provides guidance for the main fuel accelerated regulation system in design and maintenance.
\end{abstract}

\section{Introduction}

Surge is an unstable working condition under low frequency, high amplitude oscillation of airflow along compression system axial direction. As a special case that aero-engines often encounter during acceleration, severe engine surge may cause mechanical damage, loss of control and stall parking, which seriously endanger the safety of aircraft flight ${ }^{[1]}$. As an important component of air power device control system, the function of aero-engine main fuel accelerated regulation system is to keep the engine always working at design condition during acceleration through automatically and effectively regulating the fuel supply for engine, thus efficiently reduce the engine surge faults. The main fuel accelerated regulation system is complex-constructed, composed of large quantity of sensors, limiters, valves and system attachments. The more complex a system is, the higher requirement for efficient cooperation among components of system, prompting the system to present higher reliability ${ }^{[2-3]}$. Therefore, in order to ensure the aircraft flight safety, avoid catastrophic accidents and reduce the loss of personnel and property, conducting reliability analysis aiming at assessing system security and identifying system weaknesses to improve security for aero-engine main fuel accelerated regulation system has important practical significance.

In system reliability analysis, fault tree analysis(FTA) is a widely used method for large complex system, which is originally proposed by Bell Telephone Laboratory as a security assessment technique for militia emission control system. As a detailed deductive analysis method, FTA analyzes all the obvious or potential factors(including hardware, software, environment and human factors) which may or may have caused system failures, and according to certain events logical relationship to draw the logical correlation diagram(fault tree diagram), finally to determine the various possible factors combinations that caused system to take appropriate measures for improvement, and also deduce the system failure probability based on factors failure probability ${ }^{[4-5]}$.

Traditional fault tree analysis requires the exact failure probability value of bottom events or detailed failure statistics of system. However, for the large complex system like aero-engine main fuel accelerated regulation system, because the sample statistics for system components faults is usually not sufficient, it's difficult to describe bottom events faults with exact failure probability value. Therefore, considering the uncertainty of estimated value, bottom events failure probability is often treated as 
random variables subject to known probability distribution, and this process obvious lack of objectivity. Against the weakness of traditional FTA quantitative analysis method, paper[6] introduced fuzzy set theory and possibility theory into fault tree analysis method, and proposed a fault tree analysis method of hydraulic system based on arithmetic operation of trapezoidal fuzzy numbers; Paper[7] introduced triangular fuzzy number and normal fuzzy number to describe bottom events failure probability, realizing the quantitative assessment of system fault tree. But they're all related to the problem that determination of membership function has critical requirement for amount of data. Paper[8] combined dempster-shafer theory with interval probability theory, and proposed FTA interval analysis method of spaceborne antenna deployment mechanism to conduct the system functional reliability analysis.

This paper conducted fault tree analysis for aero-engine main fuel accelerated regulation system under poor failure statistics information, and proposed a reliability analysis method of aero-engine main fuel accelerated regulation system based on interval model. Firstly, according to main fuel accelerated regulation system principle and failure mechanism, the system fault tree model is established. Then interval number is introduced to describe the uncertainty of bottom event, and achieve the uncertainty transfer from bottom events to top event through constructing and/or gate interval operator. Finally, the failure probability estimation process of system top event is given based on double Monte Carlo method, and get the accurate failure interval of system. At the same time, the importance measurement analysis of bottom events is conducted to identify key failure events, which provided guidance for the main fuel accelerated regulation system in design and maintenance.

\section{Fault tree model of main fuel accelerated regulation system}

Based on system working principle, composition structure and fault classification, the system fault tree is constructed, as shown in Fig. $1^{[9]}$.

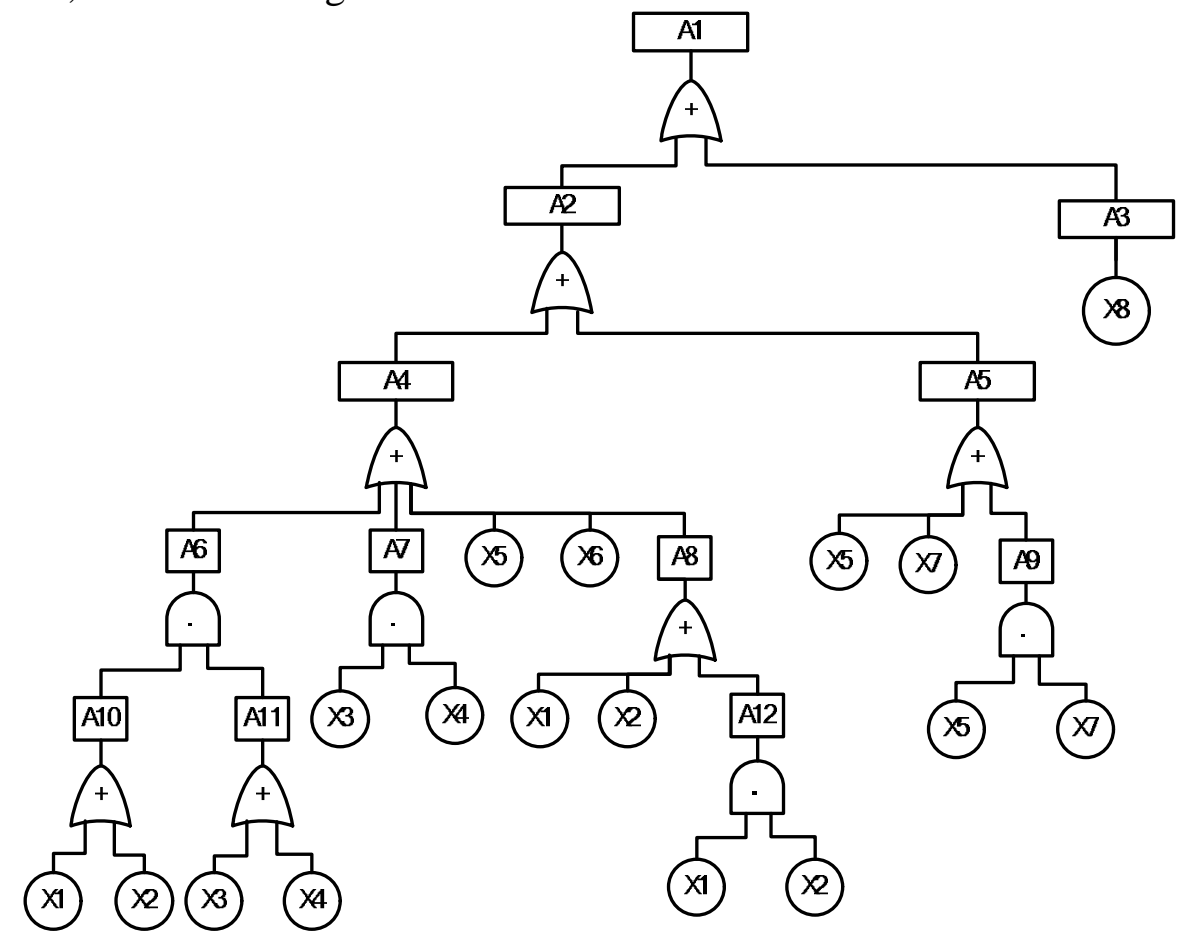

Fig.1 Fault tree of main fuel accelerated regulation system

A1-Engine surge; A2-Surge during acceleration; A3-Surge margin is too small; A4-Pressure-Increasing Limiter control acceleration time is too short; A5-Delayer control acceleration time is too short; A6-Pressure-Increasing Limiter valve closing too early; A7-Valve open strength is too small; A8-Pressure-Increasing Limiter piston right ventricle oil pressure rising too fast; A9-Delayer piston and follower piston moving left too fast; A10-Piston right ventricle oil pressure rising too fast; A11-Open strength is too small; A12-Oil pressure resistance is too small; X1-No.1 current-regulator throttling too much; X2- No.2 current-regulator throttling too much; X3-Dispenser spring 
is loose; X4-Valve wear and leak; X5-Reinfusion current regulator flow is too big; X6-Pressure-Increasing Limiter valve stuck left; X7-Delay current regulator flow is too big; X8-Blade damaged.

Based on interval model, established the fault tree logic AND operator $X_{A N D}=\prod_{i=1}^{m}\left[\frac{p_{i}}{,}, \overline{p_{i}}\right]$ and the fault tree logic OR operator $X_{O R}=[1,1]-\prod_{i=1}^{m}\left\{[1,1]-\left[\underline{p_{i}}, \overline{p_{i}}\right]\right\}$, from which we can derive the failure transfer function from bottom events to top event for main fuel accelerated regulation system, as shown in Eq.(1) .

$$
\begin{aligned}
\Phi(X)= & A_{2} \cup A_{3} \\
= & \left(A_{4} \cup A_{5}\right) \cup A_{8} \\
= & {\left[\left(A_{6} \cup A_{7} \cup X_{5} \cup X_{6} \cup A_{8}\right) \cup\left(X_{5} \cup X_{7} \cup A_{9}\right)\right] \cup X_{8} } \\
= & \left\{\left[\left(A_{10} \cap A_{11}\right) \cup\left(X_{3} \cap X_{4}\right) \cup X_{5} \cup X_{6} \cup\left(X_{2} \cup X_{1} \cup A_{12}\right)\right] \cup\left[X_{7} \cup X_{5} \cup\left(X_{5} \cap X_{7}\right)\right]\right\} \cup X_{8} \\
= & \left\{\left\{\left[\left(X_{1} \cup X_{2}\right) \cap\left(X_{3} \cup X_{4}\right)\right] \cup\left(X_{3} \cap X_{4}\right) \cup X_{5} \cup X_{6} \cup\right.\right. \\
& \left.\left.\quad\left[X_{2} \cup X_{1} \cup\left(X_{1} \cap X_{2}\right)\right]\right\} \cup\left[X_{5} \cup X_{7} \cup\left(X_{5} \cap X_{7}\right)\right]\right\} \cup X_{8}
\end{aligned}
$$

\section{Fault tree interval analysis of main fuel accelerated regulation system}

\section{Failure probability analysis of top event}

Monte Carlo method is the most basic and scope-extensive numerical simulation method, and it has no critical requirements for the form and dimension of performance function and dimension and distribution form of variables, which is easy in programming ${ }^{[10]}$. The basic idea: Conduct the sampling with the joint probability density function of basic random variables, and make the ratio of sample points number fallen in failure area and total number of sample points as the unbiased estimate of failure probability value.

Based on probability density function $f_{X}(\boldsymbol{x})$ of random variables, generate $N$ samples $\boldsymbol{x}_{j}(j=1,2, \mathrm{~L}, N)$, the ratio of samples number $N_{f}$ fallen in failure area and total samples number $N$ is supposed to be the estimate value $\hat{P}_{f}$ of failure probability, as shown in Eq.(2).

$$
\hat{P}_{f}=\frac{1}{N} \sum_{j=1}^{N} I_{F}\left(\boldsymbol{x}_{j}\right)=\frac{N_{f}}{N}
$$

where $I_{F}(x)=\left\{\begin{array}{ll}1 & x \in F \\ 0 & x \notin F\end{array}\right.$ is the indicator function of failure area.

The failure probability estimation process of main fuel accelerated regulation system based on double Monte Carlo method is given below:

(1) For $n$ bottom events failure probability interval $P_{i}(i=1 \sim n)$, generate $M$ groups of determined value $P_{j 1}, P_{j 2}, \ldots, P_{j n}(j=1 \sim M)$ subject to uniform distribution within their own probability with random number generator ( Note: In the next step (2) and (3), $j$ is determined value ) ;

(2) For each group of sampled determined value $P_{j 1}, P_{j 2}, \ldots, P_{j n}$ of bottom events failure probability, the determined value $g_{j}$ of top event failure probability can be deduced by further sampling method, and the detailed sampling process is as follows: Based on fault tree AND/OR gate logic feature, the failure transfer function from bottom events to top event can be deduced, then uniformly and randomly sample $N$ times within [0,1] to get sample $T_{k}(k=1 \sim N)$. Put $P_{j 1}$ as an example, if $T_{k} \leq P_{j 1}$ then set $Q_{k}=1$, if $T_{k}>P_{j 1}$ then set $Q_{k}=0$, then get $N$ sample points composed of 0,1 for $P_{j 1}$, and set to $X_{j 1}^{(1)}, X_{j 1}^{(2)}, \ldots, X_{j 1}^{(N)}$; Similarly we can get the sample points $X_{j i}^{(1)}, X_{j i}^{(2)}, \ldots, X_{j i}^{(N)}(i=1 \sim n)$ for other $P_{j i}(i=1 \sim n)$. Substitute $N$ groups of samples $X_{j 1}^{(k)}, X_{j 2}^{(k)}, \ldots, X_{j n}^{(k)}(k=1 \sim n)$ into failure 
transfer function, set " 1 " as failure and " 0 " as safe, then we can get $N$ sample points of top event composed of 0 and 1 , finally the top event failure probability value is $g_{j}=N_{1} / N$ ( $N_{1}$ is the number of sample value " 1 " in $N$ samples for top event)

(3) If $g_{j}>g_{2}=0$, then set $g_{2}=g_{j}$, if $g_{j}<g_{1}=1$ then set $g_{1}=g_{j}$;

(4) The step (2)、(3) circle $M$ times, and the final $g_{1}$ is supposed to be the lower bound value of top event failure probability interval, $g_{2}$ is the upper bound value, finally the failure probability interval of top event is $\left[g_{1}, g_{2}\right]$ 。

The flow diagram of failure probability estimation process for main fuel accelerated regulation system based on double Monte Carlo method is shown in Fig.2.

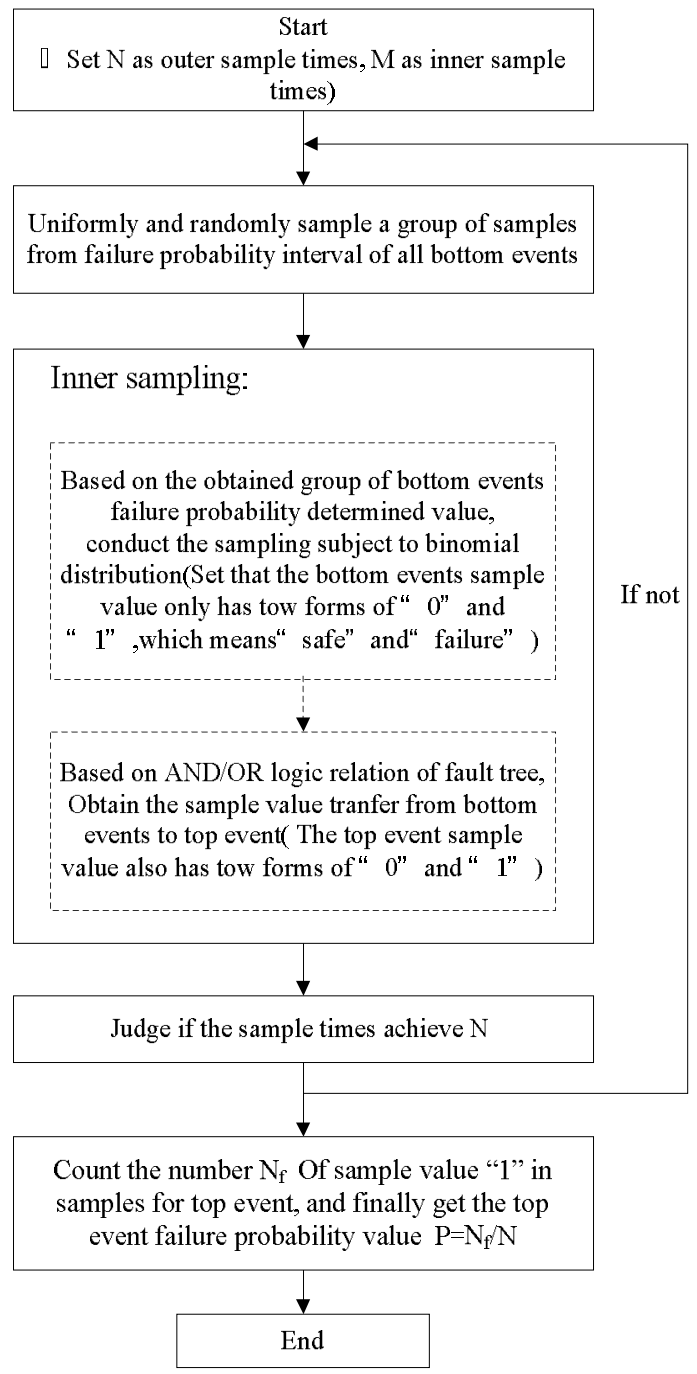

Fig.2 Flow diagram of failure probability estimation process based on double Monte Carlo method

This paper discusses the reliability of main fuel accelerated regulation system after working 10000h. Based on convergence criteria, set outer sampling times to 10000 and inner sampling times to 100000, after double Monte Carlo sampling calculation, finally we deduced that the system failure probability range of main fuel accelerated regulation system after working $10000 \mathrm{~h}$ is $[0.0154,0.0345]$.

\section{Importance measurement analysis of bottom events}

This paper also conduct the importance measurement analysis for bottom events of main fuel accelerated regulation system, including structural importance, probability importance and key importance, in order to identify key bottom events for further focus that have greater impact on system failure than other bottom events. 
Structural importance $I_{p}\left(X_{i}\right)$ reflects the increased proportion of top event states from fault to safe in all possible states of system when bottom event state change from fault to normal.

$$
I_{p}\left(X_{i}\right)=\frac{1}{2^{n-1}} N_{p}\left(X_{i}\right)
$$

where $n$ is the number of bottom events, $N_{p}\left(X_{i}\right)$ is the number of times that top event states change from fault to safe when bottom event state change from fault to normal.

Probability importance $\Delta P_{T O P}$ reflects the decreased proportion of system top event failure probability when bottom event state change from fault to normal.

$$
\Delta P_{T O P}=P_{T O P}\left(\phi\left(X_{i}\right)=1\right)-P_{T O P}\left(\phi\left(X_{i}\right)=0\right)
$$

where $\phi\left(X_{i}\right)=0$ and $\phi\left(X_{i}\right)=1$ means "fault" and "safe" of bottom event $i$.

Key importance not only reflects the effect of bottom event failure probability change on top event failure probability change, but also reflect the effect of bottom event failure probability value size on top event failure probability change. And the key importance $I_{i}^{C}(t)$ is defined as the ratio of failure probability change rate of bottom event $i$ and the top event failure probability change rate.

$$
I_{i}^{C}(t)=\lim _{\Delta P_{i}=0} \frac{\Delta G_{S}(t) / G_{S}(t)}{\Delta P_{i}(t) / P_{i}(t)}=\frac{\partial G_{S}(t)}{\partial P_{i}(t)} \cdot \frac{P_{i}(t)}{G_{S}(t)}=I_{i}^{P}(t) \cdot \frac{P_{i}(t)}{G_{S}(t)}
$$

After detailed calculation, the quantized interval of structural importance, probability importance

\begin{tabular}{|c|c|c|c|c|c|}
\hline $\begin{array}{c}\text { Bottom } \\
\text { Event No. }\end{array}$ & Probability & Importance & Structural Importance & \multicolumn{2}{|c|}{ Key Importance } \\
\hline $\mathrm{X} 1$ & {$[0.976521$,} & $0.977511]$ & {$[0.031124$,} & {$[0.172014$,} & $0.176816]$ \\
\hline $\mathrm{X} 2$ & {$[0.974227$,} & $0.978627]$ & {$[0.031014$,} & {$[0.211003$,} & $0.212422]$ \\
\hline $\mathrm{X} 3$ & {$[0.000454$,} & $0.000718]$ & {$[-0.000014,-0.000052]$} & {$[0.000002$,} & $0.000005]$ \\
\hline $\mathrm{X} 4$ & {$[-0.000012$} & $-0.000030]$ & {$[-0.000131,-0.000159]$} & {$[-0.000001$} & $-0.000002]$ \\
\hline $\mathrm{X} 5$ & {$[0.974124$,} & $0.979719]$ & {$[0.031115, \quad 0.031438]$} & {$[0.243145$, } & $0.248102]$ \\
\hline X6 & {$[0.973125$,} & $0.975824]$ & {$[0.031010$} & {$[0.101527$,} & $0.105907]$ \\
\hline $\mathrm{X} 7$ & {$[0.974625$,} & $0.979162]$ & {$[0.031102$,} & {$[0.230016$, } & $0.230249]$ \\
\hline $\mathrm{X} 8$ & {$[0.968579$,} & $0.972748]$ & {$[0.031102, \quad 0.031250]$} & {$[0.003154$,} & $0.003519]$ \\
\hline
\end{tabular}
and key importance is given below, as shown in Table 1.

Table 1 Quantized interval of all bottom events importance

From the three importance index in Table 1, it can be concluded that the sorting order of all bottom events is $\mathrm{X} 5>\mathrm{X} 7>\mathrm{X} 2>\mathrm{X} 1>\mathrm{X} 6>\mathrm{X} 8>\mathrm{X} 3>\mathrm{X} 4$, from which we can learn that bottom event $\mathrm{X} 3$, $\mathrm{X} 4$ (Dispenser spring is loose, Valve wear and leak) have the least effect on system failure, while bottom event X5, X7 (Reinfusion current regulator flow is too big, delay current regulator flow is too big) have the largest effect on system failure, which belong to weak link and should be given further attention in system design and maintenance.

\section{Conclusions}

In this paper, the interval model is introduced to effectively assess the reliability of aero-engine main fuel accelerated regulation system under poor failure statistics information:

(1) The fault tree interval analysis model of aero-engine main fuel accelerated regulation system has been established, the problem of insufficiency in probability statistics information of bottom events failure rate has been solved;

(2) The AND/OR gate interval logic operator has been constructed to realize the uncertainty transfer from bottom events to top event, and the failure probability estimation process of main fuel accelerated regulation system has been proposed based on double Monte Carlo method, finally the accurate failure probability interval of system has been obtained;

(3) The importance measurement analysis of bottom events has been conducted, and "Reinfusion current regulator flow is too big" and "Delay current regulator flow is too big" has been found to be the 
key failure bottom event, which provided guidance for the main fuel accelerated regulation system in design and maintenance.

\section{Acknowledgements}

This work was financially supported by the NPU-FFR-JC20100232, Specialized Research Fund for the Doctoral Program of Higher Education (20136102120032) and the Fundamental Research Funds for the Central Universities (3102015BJ(II )JL04).

\section{References}

[1] Luo ZM, Zheng L, Zhang YX. The research and development of aircraft surge [J]. Machine design and manufacturing engineering, 2015,44(6):1-4.

[2] Huang W, Huang XH. Adaptive acceleration control for aero-engine based on active anti-surge control [J]. Measurement and control technology, 2013,32(4):61-65.

[3] Chen HL. Modeling and simulation for performance of the mechanical-hydraulic oil system of X-8 aero-engine [D]. Xi'an: Northwestern polytechnical university, 2006.

[4] $\mathrm{Xu} \mathrm{R}$, Che JG, Yang ZB, Che XY. The fault tree analysis and its application in the system reliability analysis [J]. Command and control \& simulation, 2010,32(1):112-115.

[5] Tao YJ, Dong DC, Ren P. Uncertainty analysis of system reliability estimate based on fault tree [J]. Journal of tongji university, 2010,38(1) :141-145.

[6] Yao CY, Zhao JY. Research on fuzzy fault tree analysis method of hydraulic system [J]. China mechanical engineering, 2007,18(14):1656-1659.

[7] Li YF, Du L, Xiao NC, Huang HZ, Miao Q. Fuzzy fault tree analysis for auto drive axle system[J]. Journal of xi' an jiaotong university, 2009,43(7):110-114.

[8] Lin LG, Chen JJ. Interval analysis method of fault tree for the deployment system of a large satellite antenna [J]. Journal of mechanical strength, 2010,32(1):68-73.

[9] Ding PB. The application research of reliability analysis based on airworthiness in aero-engine surge [D]. Xi' an: Northwestern polytechnical university, 2010.

[10] Shao YF, Xue HJ. Simulation for reliability analysis based on fault tree [J]. Journal of mechanical strength, 2008,30(3):381-385. 